\title{
Genetic Diversity Analysis of Elite Doubled Haploid Rice Genotypes for Yield Attributing Traits in White Nile State, Sudan
}

\author{
Khalid A. Osman ${ }^{*}$, Sara M. Abdalla1, Kyung-Ho Kang${ }^{2}$, Lee Sang-Bok ${ }^{2}$, Ahmed E. Mohamed ${ }^{1}$, \\ Yassir M. Ahmed ${ }^{1}$, Amir I. Ismial ${ }^{1}$ \\ ${ }^{1}$ Agricultural Research Corporation (ARC), Rice Breeding Program, White Nile Research Station, Kosti, Sudan \\ ${ }^{2}$ National Institute of Crop Science, Rural Development Administration (RDA), South Korea \& AfricaRice, Saint-Louis, Senegal \\ Email: *khalrice@gmail.com
}

How to cite this paper: Osman, K.A., Abdalla, S.M., Kang, K.-H., Sang-Bok, L., Mohamed, A.E., Ahmed, Y.M. and Ismial, A.I. (2022) Genetic Diversity Analysis of Elite Doubled Haploid Rice Genotypes for Yield Attributing Traits in White Nile State, Sudan. Agricultural Sciences, 13, 330-344. https://doi.org/10.4236/as.2022.133023

Received: January 29, 2022

Accepted: March 5, 2022

Published: March 8, 2022

Copyright $\odot 2022$ by author(s) and Scientific Research Publishing Inc. This work is licensed under the Creative Commons Attribution International License (CC BY 4.0).

http://creativecommons.org/licenses/by/4.0/

\begin{abstract}
The development of new rice varieties is highly dependent on genetic diversity in desirable agronomic traits. Therefore, this study aimed to identify potential genotypes having the characters of Korean varieties (Tongil-type) and japonica developed through doubled haploid (DH) technology to apply in our breeding materials. 35 elite DH lines derived from another culture of Korean and African rice along with two local checks were planted in a randomized complete block design with three replications during the two seasons of 2019 and 2020. All evaluated genotypes exhibited a wide and significant variation in the ten measured traits. The highest heritability related to high genetic advance was recorded for the number of tiller/plant, grain yield $t / h a$, number of filled grain per panicle, and thousand grain weights (g). Genotypic coefficient of variation and genetic advance were recorded for number of tiller/plant, number of filled grain per panicle, 1000 grain weight and grain yield $t / h a$ in both seasons. Moreover, there was a highly significant and positive correlation of grain yield with number of filled grain per panicle (0.65), number of tiller/plant (0.64) and number of panicle per $\mathrm{m}^{2}(0.54)$. Cluster analysis based on grain yield components trait grouped the 37 rice genotypes into four clusters. Cluster B was the largest and consisted of 13 genotypes. Finally, it could be concluded that, based on number of productive tillers, number of filled grain/panicle, number of panicle per $\mathrm{m}^{2}$, and grain yield, the lines KF170506, KF170509, KF170542, KF170530, KF170543, KF170500 and KF170510 were high potential for further selection for new type of irrigated rice. In addition, hybridization of these 7 high-yielding could be used to achieve higher heterosis among the genotypes. Furthermore, this evaluation could be useful in developing reliable selection indices for improving rice breeding programs.
\end{abstract}




\section{Keywords}

Doubled Haploid, Anther Culture, Yield Components, Heritability and Correlation

\section{Introduction}

Rice (Oryza sativa L.) is the world's leading cereal crop as more than half of the world's population is dependent on it as their staple food [1]. The rising World population is increasing rapidly; rice production needs to be increased to meet its demands in the coming years in order to keep pace with increasing population mainly in Asia and Sub-Saharan Africa [2]. It is estimated that a 50\% increase in rice grain yield may be required by 2050 to keep hunger away [3]. This important cereal is cultivated and consumed in White Nile State in Sudan but its production is characterized by poor yields resulting from the use of low farm inputs and cultivation of unimproved cultivars with poor yield potentials [4]. Hence, improving rice yield potential or its yield under various conditions is the foremost task for rice breeders [5].

In semiarid regions, drought is the major threat to crop production, but climate change and variability often bring floods to the regions, forming seasonal wetlands causing damage to local, drought-adapted staple grains and, hence, low yields and food deficit. Introduction of the semiaquatic crop rice (Oryza spp.) to these semiarid wetlands could complement the dry land crop's low yields and overcome the food shortage problem. The most sustainable way to increase rice production is via genetic and breeding improvements [6]. Perhaps even more importantly, rice breeding enables the integration of novel traits, which is essential in achieving yield stability in the changing climatic conditions we are facing [7]. The challenge for contemporary plant breeding is not only to integrate new traits into our crops but to accelerate the genetic gain of its breeding programs at the same time, in order to achieve a doubling in speed of yield increase [8].

Another culture is a tissue culture procedure that can be applied in the breeding in order to accelerate the process of gaining pure lines [9]. With the development of doubled haploid (DH) techniques on improving the speed of genetic gain when applied in breeding programs, as well as their importance and diverse applicability in basic and applied research [10]. Another culture breeding system that can shorten varietal development cycles by 3 - 5 years allows breeders to improve existing local varieties. DH techniques have been, and are being, used to accelerate the breeding programs of a range of crops, most notably maize, wheat, rice and barley [11]. Rice researchers in South Korea have made breakthrough in yield potential occurred from modifying the existing high yield plant type to improve biomass, harvest index, reaching 7 - 8 tons/ha in polished rice. By formulated prototypes of semi-dwarf rice cultivars called "Tongil-type" (TGT) through 
wide crosses between indica and japonica cultivars by using Anther culture techniques. The desired characters of the TGT rice are compact growth, good number of productive tillers, big panicles and good grain filling (120 - 200 grains), semi-dwarf (80 - $100 \mathrm{~cm}$ in height), erect and thick leaves with dark green color, medium earliness (100 - 130 days), deep rooting system, soft and sticky endosperm and high resistance to major pests and diseases [12]. Recently, huge DH progenies were obtained from interspecific crosses between Tongil-type and Japonica with African varieties and wild species including (O. glaberrima, O. longistaminata, and $O$. rufipogon). Many of them were distributed to different African countries in order to determine the agronomic character stabilities for a further selection of desirable characters, particularly in terms of yield and yield components. This will be serving as good resources for improving yield potential of local varieties, particularly in Sudan.

The magnitude of genetic variability provides useful information with regard to the possibility and extent of improvement that may be expected in the characters through breeding and selection. Breeders are interested in evaluating genetic diversity based on morphological characteristics as they are inexpensive, rapid, and simple to score. Moreover, this evaluation could be useful in developing reliable selection indices for important agronomic traits in rice. Therefore, the main objective of the present investigation was to identify the existing genetic diversity and to select high yielding $\mathrm{DH}$ lines to cultivate under aerobic condition with the characters of the Tongil-type. That can establish relationship in yields and their components and utilization of the available population in future rice breeding programs.

\section{Materials and Methods}

\subsection{Study Site and Experimental Design}

A total of 172 doubled haploid lines, were produced through another culture of the (F2-F3) generations derived from interspecific crosses among Korean Tongil-type (Semi-dwarfism, high-yielding potential with medium amylose and short grain) and Japonica (Earliness, low-medium amylose, soft and sticky endosperm) with African varieties and wild species (high biomass, disease resistance and low yield) were established at (Tissue culture laboratory Africa-Rice St. Louis). Of these doubled haploid lines were grown at Sahel Station in nursery test observation then after several series of selection and fixation, in advanced generation, 172 potential lines with the required adaptive traits were received in season 2018 and conducted in Observation Yield Trial; then again selected the superior $35 \mathrm{DH}$ lines along with two checks namely Sahel177 and Arica3, were grown for two consecutive seasons (2019 and 2020) during the rainy season under irrigated condition; at White Nile Research Station Farm (latitude $14^{\circ} 24^{\prime} \mathrm{N}$ and longitude $33^{\circ} 22^{\prime} \mathrm{E}$ ), Kosti of the Agricultural Research Corporation (ARC) Sudan. The soil of the experimental plots was classified as vertisol with high clay content ( $40 \%$ to $65 \%)$, less than $1 \%$ organic carbon, low in available nitrogen 
$\left(0.03 \%\right.$ total nitrogen) and medium in available $\mathrm{P}_{2} \mathrm{O}_{5}$ (406 to $700 \mathrm{ppm}$ total phosphorus), $\mathrm{pH}$ values is slightly alkaline which ranging from 7 to 8.2. The climate was semi-arid (Table 1). The experimental plots were laid out in randomized complete block design (RCBD) with three replications. In both seasons deep plough, harrowing and leveling were practiced to prepare experimental area. The seeds were drilled on July $7^{\text {th }}$ and $5^{\text {th }}$ of 2019 and 2020 in turn, using a seed rate of $80 \mathrm{~kg} / \mathrm{ha}$. The plot size was $(1 \mathrm{~m} \times 6 \mathrm{~m})$ with $0.2 \mathrm{~m}$ apart consisted 5 rows giving a total area of $6 \mathrm{~m}^{2}$. Recommended fertilizer of Urea and Triple Super Phosphate (TSP) are at the rate of $129 \mathrm{~kg}$ of Nitrogen $\left(\mathrm{N}_{2}\right)$ per hectare and 43 $\mathrm{kg}$ of passphrase $\left(\mathrm{P}_{2} \mathrm{O}_{5}\right)$ per hectare respectively. TSP was applied as basal dose during final land preparation. Nitrogen was top-dressed in two equal split doses at 21 days after sowing and the other before panicle initiation. Hand weeding was performed three times per season. All plots were irrigated at sowing and then at weekly intervals until it reached the flowering stage and then every 3 days. All other agronomic practices were applied as per the recommendation for rice production in the two seasons to raise a healthy rice crop.

\subsection{Data Collection}

Data were collected on days to flowering (days from sowing to time when the $50 \%$ of the plants were flowering) and days to maturity (days from sowing to time when $80 \%$ of the panicles reached full maturity). Then at harvest, plant height $(\mathrm{cm})$, panicle length $(\mathrm{cm})$, number of tiller/plant, number of filled grain/panicle, percent of unfilled grain/panicle, 1000 grain weight $(\mathrm{g})$ and grain yield $(\mathrm{t} / \mathrm{ha})$ based on grain yield per plot were recorded. The data were collected according to standard evaluation systems for rice standard evaluation system (SES) [13]. Ten randomly selected plants in the net harvested area of each plot were used for data collection.

\subsection{Statistical Analysis}

Analysis of variance (ANOVA) was carried out on the data to assess the genotypic effects and their interaction using the general linear model (GLM) procedure for randomized complete blocks design in [14] SAS (version 9.1). Estimates of variance components were generated and combined analysis of variance was done for the traits in which the mean squares were homogenous. The phenotypic and genotypic variances and their coefficients, heritability in the broad sense and genetic advance were computed according to the formula described by [15]. Combined over seasons were used to compute simple linear correlation coefficients., between grain yields and nine other traits.

\section{Results and Discussion}

\subsection{The Genetic Analysis of the Traits}

The results showed that there is a presence of an acceptable amount of diversity 
Table 1. Name of 35 doubled haploid rice genotypes plus two checks used in the present study.

\begin{tabular}{|c|c|c|c|c|}
\hline SN & Designation & Pedigree & Ecology & Origin \\
\hline 1 & KF170495 & SR35266-2-11-1-1 & Irrigated & AfricaRice/KAFACI \\
\hline 2 & KF170399 & SR35278-1-7-3-2 & Irrigated & AfricaRice/KAFACI \\
\hline 3 & KF170506 & SR35266-2-17-2-1 & Irrigated & AfricaRice/KAFACI \\
\hline 4 & KF170540 & SR34590-HB3433-7-1-1 & Irrigated & AfricaRice/KAFACI \\
\hline 5 & KF170409 & SR35278-1-9-3-3 & Irrigated & AfricaRice/KAFACI \\
\hline 6 & KF170544 & SR34590-HB3433-8-2-1 & Irrigated & AfricaRice/KAFACI \\
\hline 7 & KF170410 & SR35278-2-8-2-1 & Irrigated & AfricaRice/KAFACI \\
\hline 8 & KF170406 & SR35278-1-9-1-3 & Irrigated & AfricaRice/KAFACI \\
\hline 9 & KF170489 & SR35266-2-7-3-1 & Irrigated & AfricaRice/KAFACI \\
\hline 10 & KF170365 & SR34053(\#5-52)-1-4-2-10-3-1 & Irrigated & AfricaRice/KAFACI \\
\hline 11 & KF170511 & SR35266-2-19-1-1 & Irrigated & AfricaRice/KAFACI \\
\hline 12 & KF170496 & SR35266-2-11-4-1 & Irrigated & AfricaRice/KAFACI \\
\hline 13 & KF170530 & SR34590-HB3433-2-2-1 & Irrigated & AfricaRice/KAFACI \\
\hline 14 & KF170482 & SR35266-2-5-3-1 & Irrigated & AfricaRice/KAFACI \\
\hline 15 & KF170500 & SR35266-2-12-4-1 & Irrigated & AfricaRice/KAFACI \\
\hline 16 & KF170488 & SR35266-2-7-2-1 & Irrigated & AfricaRice/KAFACI \\
\hline 17 & KF170405 & SR35278-1-9-1-2 & Irrigated & AfricaRice/KAFACI \\
\hline 18 & KF170516 & SR35266-2-20-3-1 & Irrigated & AfricaRice/KAFACI \\
\hline 19 & KF170543 & SR34590-HB3433-8-1-1 & Irrigated & AfricaRice/KAFACI \\
\hline 20 & KF170217 & SR34054-1-21-4-3-1-1 & Irrigated & AfricaRice/KAFACI \\
\hline 21 & KF170501 & SR35266-2-12-5-1 & Irrigated & AfricaRice/KAFACI \\
\hline 22 & KF170484 & SR35266-2-6-1-1 & Irrigated & AfricaRice/KAFACI \\
\hline 23 & KF170505 & SR35266-2-17-1-1 & Irrigated & AfricaRice/KAFACI \\
\hline 24 & KF170542 & SR34590-HB3433-7-3-1 & Irrigated & AfricaRice/KAFACI \\
\hline 25 & KF170485 & SR35266-2-6-2-1 & Irrigated & AfricaRice/KAFACI \\
\hline 26 & KF170498 & SR35266-2-12-2-1 & Irrigated & AfricaRice/KAFACI \\
\hline 27 & KF170407 & SR35278-1-9-3-1 & Irrigated & AfricaRice/KAFACI \\
\hline 28 & KF170509 & SR35266-2-18-2-1 & Irrigated & AfricaRice/KAFACI \\
\hline 29 & KF170390 & SR35276-2-4-3-2 & Irrigated & AfricaRice/KAFACI \\
\hline 30 & KF170532 & SR34590-HB3433-4-1-1 & Irrigated & AfricaRice/KAFACI \\
\hline 31 & KF170502 & SR35266-2-16-1-1 & Irrigated & AfricaRice/KAFACI \\
\hline 32 & KF170504 & SR35266-2-16-3-1 & Irrigated & AfricaRice/KAFACI \\
\hline 33 & KF170503 & SR35266-2-16-2-1 & Irrigated & AfricaRice/KAFACI \\
\hline 34 & KF170508 & SR35266-2-18-1-1 & Irrigated & AfricaRice/KAFACI \\
\hline 35 & KF170510 & SR35266-2-18-3-1 & Irrigated & AfricaRice/KAFACI \\
\hline 36 & Sahel177 & Sahel177 (Check) & Irrigated & Senegal \\
\hline 37 & Arica3 & Arica3 (Check) & Irrigated & Senegal \\
\hline
\end{tabular}


among the genotypes. This gives an opportunity for rice breeders to improve those traits through selection and hybridization to improve the desired traits. The range and mean of genotypes for all studied traits also indicated wide ranges of variation which also revealed a possible amount of variability among the genotypes (Table 2).

The broad-sense heritability is the relative magnitude of genotypic and phenotypic variances for the traits and it is used as a predictive role in selection procedures. This gives an idea of the total variation ascribable to genotypic effects, which are an exploitable portion of the variation. The characters that showed relatively high heritability estimates $(\geq 60 \%)$ were days to flowering $(90.3$ and 64.1), grain yield $\mathrm{t} / \mathrm{ha}$ (89.9 and 77.7), number of filled grain/panicle (84.0 and 85.8), panicle length (82.5 and 61.3) and number of tiller/plant (73.1 and 86.1) in both seasons respectively, indicating the optimizing of homogeneous of these lines and their improvement could be achieved by mass selection. Similar results were reported by [16]. This indicated that the selection of these traits would be more effective as compared to others. The moderate heritability estimate for grain yield was attributed to the fact that yield is a complex trait and is controlled by many genes. Since high heritability does not always indicate high genetic gain. Heritability with genetic advance considered together should be used in predicting the ultimate effect for selecting superior varieties [2] and [17]. High genetic advance and heritability were recorded for the number of tiller/plant number of filled grain/panicle 1000 grain weight and grain yield $t / h a$. These results suggested that these traits were primarily under genetic control and selection for these traits can be achieved through their phenotypic performance. High heritability estimates with low genetic advance observed for days to maturity, number of unfilled grain/panicle and panicle $/ \mathrm{m}^{2}$ indicated non-additive type of gene action and that genotype $x$ environment interaction played a significant role in the expression of the traits. The genetic analysis of quantitative traits is a prerequisite for plant breeding programs, which can lead to a systemic method of design and to the appropriate planning of plant breeding strategies. The current study suggests that the PCV was higher than the GCV for all traits. This was also the case for all the traits observed in another study; [4] reported that the environmental effect on any trait is indicated by the magnitude of the differences between the genotypic and phenotypic coefficients of variation; large differences reflect a large environmental effect, whereas small differences reveal a high genetic influence. In this study, the small differences between the PCV and GCV for most of the traits, such as number of tiller/plant, number of filled grain per panicle, 1000 grain weight and grain yield $t / h a$, represented some degree of environmental influence on the phenotypic expression of these characters. It also suggests that selection based on these characters would be effective for future crossing programs. The other traits, which showed a higher difference between PCV and GCV, indicated that the environmental effect on the expression of those traits is higher and that selection based on these characters independently is not effective for further yield improvement. 
Table 2. Estimates of phenotypic variability, heritability in broad sense (h2B), genotypic (GCV) and phenotypic (PCV), coefficients of variation, genetic advance as percentage of the mean for 10 traits in 37 doubled haploid (DH) rice genotypes grown at the WNRS Farm, seasons 2019 and 2020.

\begin{tabular}{|c|c|c|c|c|c|c|c|c|c|}
\hline Variable & Seasons & Mean & Minimum & Maximum & F value & GCV\% & PCV\% & $\mathrm{h} 2 \mathrm{~b} \%$ & GAM\% \\
\hline \multirow{2}{*}{ Days to flowering } & 2019 & $94.20 \pm 0.6$ & 80 & 111 & $27.92^{\star *}$ & 59.54 & 62.66 & 90.3 & 1.97 \\
\hline & 2020 & $101.61 \pm 0.46$ & 89 & 111 & $6.35^{\star *}$ & 39.49 & 49.33 & 64.1 & 1.30 \\
\hline \multirow{2}{*}{ Days to maturity } & 2019 & $115.9 \pm 0.3$ & 110 & 124 & $6.82^{* *}$ & 25.75 & 31.70 & 66.0 & 1.17 \\
\hline & 2020 & $120.15 \pm 0.34$ & 108 & 127 & $9.05^{\star *}$ & 28.77 & 33.71 & 72.9 & 1.25 \\
\hline \multirow{2}{*}{ Plant height $(\mathrm{cm})$} & 2019 & $83.6 \pm 0.7$ & 63.5 & 104.3 & $6.38^{\star *}$ & 60.52 & 75.52 & 64.2 & 1.58 \\
\hline & 2020 & $86.9 \pm 0.58$ & 63.9 & 107.4 & $10.81^{\star * *}$ & 58.48 & 66.83 & 76.6 & 1.82 \\
\hline \multirow{2}{*}{ Panicle length $(\mathrm{cm})$} & 2019 & $22.1 \pm 0.2$ & 16.7 & 26.8 & $15.18^{\star *}$ & 34.95 & 38.47 & 82.5 & 7.70 \\
\hline & 2020 & $22.2 \pm 0.16$ & 18.3 & 26.2 & $5.76^{* *}$ & 28.35 & 36.20 & 61.3 & 5.70 \\
\hline \multirow{2}{*}{ Number of tiller/plant } & 2019 & $4.8 \pm 0.1$ & 3 & 6 & $9.17^{\star}$ & 32.69 & 38.22 & 73.1 & 30.80 \\
\hline & 2020 & $4.9 \pm 0.10$ & 3 & 8 & $19.64^{\star \star}$ & 42.55 & 45.84 & 86.1 & 35.62 \\
\hline \multirow{2}{*}{ Number of panicle $/ \mathrm{m}^{2}$} & 2019 & $311.5 \pm 5.9$ & 209 & 481 & $8.59^{\star}$ & 302.4 & 357.2 & 71.7 & 0.47 \\
\hline & 2020 & $322.6 \pm 5.7$ & 215 & 489 & $7.39^{* *}$ & 276.5 & 335.1 & 68.1 & 0.43 \\
\hline \multirow{2}{*}{$\begin{array}{l}\text { Number of filled } \\
\text { grain/panicle }\end{array}$} & 2019 & $14.4 \pm 0.4$ & 8 & 25 & $16.78^{\star \star}$ & 89.91 & 98.08 & 84.0 & 11.99 \\
\hline & 2020 & $14.4 \pm 0.43$ & 8 & 32 & $19.13^{\star *}$ & 113.43 & 122.46 & 85.8 & 12.31 \\
\hline \multirow{2}{*}{$\begin{array}{l}\text { Number of unfilled } \\
\text { grain/panicle }\end{array}$} & 2019 & $106.2 \pm 1.2$ & 74 & 142 & $8.25^{\star * *}$ & 103.95 & 123.62 & 70.7 & 1.37 \\
\hline & 2020 & $107.1 \pm 1.3$ & 83 & 149 & $11.55^{\star *}$ & 116.68 & 132.23 & 77.9 & 1.50 \\
\hline \multirow{2}{*}{1000 grain weight (g) } & 2019 & $22.7 \pm .2$ & 15.2 & 29.2 & $7.02^{\star * *}$ & 32.64 & 39.95 & 66.7 & 6.04 \\
\hline & 2020 & $22.2 \pm 0.18$ & 18.9 & 27.9 & $74.53^{\star *}$ & 41.85 & 42.70 & 96.1 & 8.92 \\
\hline \multirow{2}{*}{ Grain yield ton/hectare } & 2019 & $6.51 \pm 0.1$ & 3.42 & 8.82 & $27.66^{\star \star}$ & 39.98 & 42.17 & 89.9 & 28.36 \\
\hline & 2020 & $6.37 \pm 0.2$ & 3.89 & 8.89 & $11.44^{\star *}$ & 34.25 & 38.86 & 77.7 & 25.12 \\
\hline
\end{tabular}

${ }^{*}, * *, * *$ Significant at $0.05,0.01$ and 0.001 probability levels, respectively.

The highest PCV was recorded for number of unfilled grain/panicle (123.62 132.23) and numbers of filled grains/panicle (98.02 - 122.4) in both seasons were also recorded by the following researchers ([18] and [19]).

\subsection{Quantitative Characteristics}

The results further revealed that most of the traits exhibited wide range of variability (Table 3(a), Table 3(b)). The late flowering genotypes were KF170390, KF170502, KF170504 and KF170496 while, the early flowering genotypes were KF170399, KF170505 and KF170510. The late maturity genotypes were KF170390, KF170504 while the early maturity genotypes were KF170405 and KF170543. Generally, most of the genotypes showed early maturing period less than 120 days. This suggested the chance of selecting earliness genotypes which can escape terminal moisture and drought stress. The range for plant height was 
Table 3. (a) Mean of growth aspects of 35 doubled haploid lines and two checks grown at White Nile Research Station Farm (WNRSF) combined over two seasons, 2019 and 2020. (b) Mean of growth aspects of 35 doubled haploid lines and two checks grown at White Nile Research station Farm (WNRSF) combined over two seasons, 2019 and 2020.

(a)

\begin{tabular}{|c|c|c|c|c|c|}
\hline Name & DF & $\mathrm{DM}$ & PLH & PL & NTP \\
\hline KF170495 & $93.8^{\mathrm{MNO}}$ & $118.5^{\mathrm{DEFG}}$ & $82.1^{\mathrm{IJKLMN}}$ & $19.4^{\mathrm{P}}$ & $5.0^{\mathrm{DE}}$ \\
\hline KF170399 & $88.8^{\mathrm{P}}$ & $116.8^{\mathrm{FGHIJ}}$ & $92.4^{\mathrm{BCD}}$ & $23.3^{\mathrm{CDEF}}$ & $5.8^{\mathrm{B}}$ \\
\hline KF170506 & $98 \mathrm{I}^{\mathrm{JKL}}$ & $116.5^{\mathrm{GHIJ}}$ & $86.6^{\mathrm{FGH}}$ & $23.9^{\mathrm{BCD}}$ & $6.5^{\mathrm{A}}$ \\
\hline KF170540 & $96.3^{\mathrm{LM}}$ & $118.8^{\mathrm{CDEF}}$ & $92.1^{\mathrm{BCDE}}$ & $22.6^{\mathrm{EFHIG}}$ & $4.8^{\mathrm{EF}}$ \\
\hline KF170409 & $102.3^{\mathrm{CDEF}}$ & $118.7^{\mathrm{DEFG}}$ & $88.1^{\mathrm{DEFG}}$ & $22.5^{\mathrm{EFHIG}}$ & $4.3^{\mathrm{GH}}$ \\
\hline KF170544 & $97.8^{\mathrm{KL}}$ & $119.3^{\mathrm{BCDE}}$ & $85.4^{\mathrm{FGHIJK}}$ & $22.5^{\mathrm{EFHIG}}$ & $3.8^{\mathrm{I}}$ \\
\hline KF170410 & $94.5^{\mathrm{MNO}}$ & $118.7^{\mathrm{DEFG}}$ & $85.5^{\text {FGHIJ }}$ & $20.1^{\mathrm{OP}}$ & $5.8^{\mathrm{B}}$ \\
\hline KF170406 & $94.8^{\mathrm{MNO}}$ & $117.5^{\mathrm{EFGHI}}$ & $81.9^{\text {JKLMN }}$ & $22.3^{\text {FHIG }}$ & $4.3^{\mathrm{GH}}$ \\
\hline KF170489 & $98.0^{\mathrm{HIJKL}}$ & $118.7^{\mathrm{DEF}}$ & $74.5^{\mathrm{OP}}$ & $21.4^{\mathrm{KLM}}$ & $4.3^{\mathrm{GH}}$ \\
\hline KF170365 & $98.7^{\mathrm{HIJKL}}$ & $118.8^{\mathrm{CDEF}}$ & $77.6^{\mathrm{OP}}$ & $24.1^{\mathrm{BC}}$ & $5.8^{\mathrm{B}}$ \\
\hline KF170511 & $102.0^{\mathrm{CDEFG}}$ & $119.8^{\mathrm{BCD}}$ & $80.2^{\mathrm{MNOP}}$ & $21.9^{\mathrm{HIJKL}}$ & $5.8^{\mathrm{B}}$ \\
\hline KF170496 & $104.0^{\mathrm{BCD}}$ & $117.5^{\mathrm{EFGHI}}$ & $82.2^{\mathrm{IJKLM}}$ & $20.9^{\mathrm{LMNO}}$ & $4.0^{\mathrm{HI}}$ \\
\hline KF170530 & $101.5^{\mathrm{DEFGH}}$ & $119.8^{\mathrm{BCD}}$ & $82.9^{\mathrm{HIJKM}}$ & $21.2^{\mathrm{KLMNO}}$ & $5.6^{\mathrm{BC}}$ \\
\hline KF170482 & $96 \mathrm{~L}^{\mathrm{MN}}$ & $117.5^{\mathrm{EFGHI}}$ & $85.2^{\mathrm{FGHIJK}}$ & $22.1^{\mathrm{HIJ}}$ & $4.8^{\mathrm{EF}}$ \\
\hline KF170500 & $100.8^{\mathrm{EFGH}}$ & $121.0^{\mathrm{ABC}}$ & $87.8^{\mathrm{EFG}}$ & $22.6^{\mathrm{EFHIG}}$ & $5.6^{\mathrm{BC}}$ \\
\hline KF170488 & $99.3^{\mathrm{GHIJK}}$ & $115.5^{\mathrm{IJKL}}$ & $82.8^{\mathrm{IJKLMN}}$ & $20.9^{\text {KLMNO }}$ & $4.0^{\mathrm{HI}}$ \\
\hline KF170405 & $97.8^{\mathrm{KL}}$ & $113.5^{\mathrm{L}}$ & $81.5^{\mathrm{IJKLMN}}$ & $21.2^{\mathrm{KLMN}}$ & $4.8^{\mathrm{EF}}$ \\
\hline KF170516 & $94.8^{\mathrm{MNO}}$ & $116.5^{\mathrm{GHIJ}}$ & $85.7^{\mathrm{FGHIJ}}$ & $21.6^{\mathrm{KLM}}$ & $4.8^{\mathrm{EF}}$ \\
\hline KF170543 & $93.8^{\mathrm{MNO}}$ & $113.3^{\mathrm{L}}$ & $87.5^{\mathrm{FG}}$ & $23.8^{\mathrm{BCD}}$ & $5.6^{\mathrm{BC}}$ \\
\hline KF170217 & $93.8^{\mathrm{MNO}}$ & $116.5^{\mathrm{GHIJ}}$ & $93.1^{\mathrm{B}}$ & $21.7^{\mathrm{KLM}}$ & $5.3^{\mathrm{CD}}$ \\
\hline KF170501 & $93.3^{\mathrm{NO}}$ & $114.0^{\mathrm{KL}}$ & $83.6^{\mathrm{GHIJKL}}$ & $24.8^{\mathrm{AB}}$ & $5.3^{\mathrm{CD}}$ \\
\hline KF170484 & $93.3^{\mathrm{NO}}$ & $115.0^{\mathrm{JKL}}$ & $81.2^{\mathrm{IJKLMNO}}$ & $22.1^{\mathrm{HIJK}}$ & $4.0^{\mathrm{HI}}$ \\
\hline KF170505 & $92.8^{\mathrm{O}}$ & $117.3^{\mathrm{EFGHI}}$ & $86.2^{\mathrm{FGHI}}$ & $20.7^{\mathrm{MNO}}$ & $4.0^{\mathrm{HI}}$ \\
\hline KF170542 & $100.0^{\mathrm{EFGHIJ}}$ & $119.3^{\mathrm{BCDE}}$ & $89.2^{\mathrm{BCDE}}$ & $22.1^{\mathrm{HIJK}}$ & $5.0^{\mathrm{CD}}$ \\
\hline KF170485 & $100.2^{\mathrm{EFGHI}}$ & $117.8^{\mathrm{DEFGH}}$ & $88.4^{\mathrm{CDEF}}$ & $21.8^{\mathrm{KLM}}$ & $5.0^{\mathrm{DE}}$ \\
\hline KF170498 & $96.1 \mathrm{~L}^{\mathrm{MN}}$ & $117.3^{\mathrm{EFGHI}}$ & $86.3^{\mathrm{FGHI}}$ & $20.9^{\mathrm{LMNO}}$ & $4.8^{\mathrm{EF}}$ \\
\hline KF170407 & $98.8^{\mathrm{HIJKL}}$ & $117.5^{\mathrm{EFGHI}}$ & $82.3^{\mathrm{IJKLMN}}$ & $23.6^{\mathrm{CDE}}$ & $5.3^{\mathrm{CD}}$ \\
\hline KF170509 & $96.6^{\mathrm{KLM}}$ & $119.3^{\mathrm{BCDE}}$ & $88.2^{\mathrm{CDEF}}$ & $23.9^{\mathrm{BCD}}$ & $6.6^{\mathrm{A}}$ \\
\hline KF170390 & $108.1^{\mathrm{A}}$ & $122.3^{\mathrm{A}}$ & $92.1^{\mathrm{BCDE}}$ & $22.9^{\mathrm{DEFGH}}$ & $4.0^{\mathrm{HI}}$ \\
\hline KF170532 & $99.5^{\mathrm{EFGHIJ}}$ & $115.8^{\mathrm{HIJK}}$ & $87.4^{\mathrm{FG}}$ & $20.2^{\mathrm{NOP}}$ & $4.0^{\mathrm{HI}}$ \\
\hline KF170502 & $105.3^{\mathrm{AB}}$ & $118.8^{\mathrm{CDEF}}$ & $92.5^{\mathrm{C}}$ & $20.8^{\mathrm{MNO}}$ & $3.6^{\mathrm{I}}$ \\
\hline KF170504 & $104.6^{\mathrm{BC}}$ & $122.3^{\mathrm{A}}$ & $81.1^{\text {KLMNO }}$ & $21.3^{\mathrm{KLM}}$ & $4.5^{\mathrm{FG}}$ \\
\hline KF170503 & $96.2^{\mathrm{LMN}}$ & $118.3^{\mathrm{DEFG}}$ & $81.0^{\text {KLMNO }}$ & $21.9^{\mathrm{HIJKL}}$ & $4.3^{\mathrm{GH}}$ \\
\hline
\end{tabular}


Continued

\begin{tabular}{cccccc}
\hline KF170508 & $94.1^{\mathrm{MNO}}$ & $118^{\mathrm{DEFGH}}$ & $79.2^{\mathrm{NOP}}$ & $21.1^{\mathrm{KLMNO}}$ & $3.8^{\mathrm{I}}$ \\
KF170510 & $92.8^{\mathrm{O}}$ & $119.8^{\mathrm{BCD}}$ & $81.4^{\mathrm{IJKLMN}}$ & $23.1^{\mathrm{CDEFG}}$ & $5.8^{\mathrm{B}}$ \\
SAHEL177 & $100.8^{\mathrm{EFGHI}}$ & $119.3^{\mathrm{BCDE}}$ & $76.0^{\mathrm{OP}}$ & $21.4^{\mathrm{KLM}}$ & $4.6^{\mathrm{EFG}}$ \\
ARICA3 & $102.8^{\mathrm{BCDE}}$ & $121.3^{\mathrm{AB}}$ & $103.0^{\mathrm{A}}$ & $25.5^{\mathrm{A}}$ & $6.0^{\mathrm{B}}$ \\
Mean & $\mathbf{9 7 . 9 \pm 0 . 4 4}$ & $\mathbf{1 1 8 . 0 \pm 0 . 3}$ & $\mathbf{8 5 . 2} \pm \mathbf{0 . 5}$ & $\mathbf{2 2 . 1} \pm \mathbf{0 . 2}$ & $\mathbf{4 . 9} \pm \mathbf{0 . 1}$ \\
CV\% & 2.5 & 1.7 & 4.5 & 4.2 & $\mathbf{8 . 3}$ \\
\hline
\end{tabular}

*, ${ }^{* *}$ significant at 0.05 and 0.01 probability respectively. Days to $50 \%$ heading (DH); Days to maturity (DM); Plant height centimeter (PLH); Panicle length centimeter (PL); No of tiller/plant (NTP).

(b)

\begin{tabular}{|c|c|c|c|c|c|}
\hline Name & NPM & NFGP & NUFGP & TGW & GY th \\
\hline KF170495 & $319^{\mathrm{FGHIJ}}$ & $104^{\mathrm{EFGH}}$ & $16^{\mathrm{DEFG}}$ & $24.3^{\mathrm{BCD}}$ & $6.92^{\mathrm{DE}}$ \\
\hline KF170399 & $369^{\mathrm{BCD}}$ & $116^{\mathrm{B}}$ & $12^{\mathrm{HIJK}}$ & $25.9^{\mathrm{A}}$ & $7.11^{\mathrm{CDE}}$ \\
\hline KF170506 & $429^{\mathrm{A}}$ & $134^{\mathrm{A}}$ & $9^{\mathrm{LMN}}$ & $23.8^{\mathrm{DE}}$ & $8.35^{\mathrm{A}}$ \\
\hline KF170540 & $277^{\mathrm{LMNO}}$ & $106^{\mathrm{DDEFG}}$ & $19^{\mathrm{C}}$ & $22.7^{\mathrm{FGH}}$ & $5.99^{\mathrm{GHI}}$ \\
\hline KF170409 & $306^{\mathrm{IJKLM}}$ & $103^{\mathrm{EFGH}}$ & $14^{\mathrm{FGHI}}$ & $19.4^{\mathrm{Q}}$ & $6.75^{\mathrm{EF}}$ \\
\hline KF170544 & $256^{\mathrm{QRS}}$ & $95^{\text {JKLMN }}$ & $22^{\mathrm{B}}$ & $20.8^{\mathrm{NOP}}$ & $5.72^{\mathrm{HIJ}}$ \\
\hline KF170410 & $357^{\mathrm{CDEF}}$ & $114^{\mathrm{B}}$ & $12^{\mathrm{HIJK}}$ & $20.5^{\mathrm{OP}}$ & $7.05^{\mathrm{CDE}}$ \\
\hline KF170406 & $332^{\mathrm{EFGHI}}$ & $110^{\mathrm{BCDE}}$ & $18^{\mathrm{CD}}$ & $21.6^{\mathrm{IJKLM}}$ & $6.21^{\mathrm{G}}$ \\
\hline KF170489 & $275^{\mathrm{NOPQ}}$ & $101^{\mathrm{GHIJK}}$ & $18^{\mathrm{CDE}}$ & $23.6^{\mathrm{DEF}}$ & $6.29^{\mathrm{G}}$ \\
\hline KF170365 & $360^{\mathrm{CDE}}$ & $116^{\mathrm{B}}$ & $11^{\mathrm{JLLM}}$ & $21.8^{\mathrm{GHIJK}}$ & $7.21^{\mathrm{BCDE}}$ \\
\hline KF170511 & $344^{\mathrm{DEFGH}}$ & $105^{\mathrm{DEFG}}$ & $11^{\mathrm{JKLM}}$ & $22.3^{\mathrm{GHIJK}}$ & $7.07^{\mathrm{CDE}}$ \\
\hline KF170496 & $272^{\mathrm{NOPQ}}$ & $94^{\text {KLMN }}$ & $14^{\mathrm{FGHI}}$ & $21.5^{\mathrm{JLLMN}}$ & $5.56^{\mathrm{IJKL}}$ \\
\hline KF170530 & $315^{\mathrm{HIJK}}$ & $117^{\mathrm{B}}$ & $15^{\mathrm{EFGH}}$ & $25.2^{\mathrm{AB}}$ & $7.45^{\mathrm{BC}}$ \\
\hline KF170482 & $300^{\mathrm{IJKLM}}$ & $114^{\mathrm{BC}}$ & $14^{\mathrm{FGHI}}$ & $23.1^{\mathrm{EFG}}$ & $6.85^{\mathrm{DE}}$ \\
\hline KF170500 & $345^{\mathrm{DEFG}}$ & $116^{\mathrm{B}}$ & $15^{\mathrm{EFGH}}$ & $22.4^{\mathrm{GHIJ}}$ & $7.30^{\mathrm{BCD}}$ \\
\hline KF170488 & $228^{\mathrm{RS}}$ & $99^{\mathrm{HIJK}}$ & $17^{\mathrm{DEF}}$ & $21.7^{\mathrm{IJKLM}}$ & $5.18^{\mathrm{L}}$ \\
\hline KF170405 & $310^{\mathrm{IIKL}}$ & $103^{\mathrm{EFGH}}$ & $16^{\mathrm{DEFG}}$ & $20.3^{\mathrm{PQ}}$ & $6.04^{\mathrm{GH}}$ \\
\hline KF170516 & $316^{\mathrm{HIJK}}$ & $102^{\mathrm{GHIJ}}$ & $12^{\mathrm{HIJK}}$ & $21.3^{\mathrm{LMNO}}$ & $6.26^{\mathrm{G}}$ \\
\hline KF170543 & $360^{\mathrm{CDE}}$ & $115^{\mathrm{B}}$ & $9^{\mathrm{LMN}}$ & $22.5^{\mathrm{GHI}}$ & $7.40^{\mathrm{BC}}$ \\
\hline KF170217 & $354^{\mathrm{DEFG}}$ & $113^{\mathrm{BCD}}$ & $8^{\mathrm{NO}}$ & $24.3^{\mathrm{BCD}}$ & $7.16^{\mathrm{BCDE}}$ \\
\hline KF170501 & $370^{\mathrm{BCD}}$ & $117^{\mathrm{B}}$ & $9^{\mathrm{LMN}}$ & $23.8^{\mathrm{DE}}$ & $7.19^{\mathrm{BCDE}}$ \\
\hline KF170484 & $291^{\text {KLMN }}$ & $94^{\text {KLMN }}$ & $17^{\mathrm{DEF}}$ & $24.1^{\mathrm{CD}}$ & $5.67^{\mathrm{HIJ}}$ \\
\hline KF170505 & $320^{\mathrm{FGHIJ}}$ & $89^{\mathrm{MN}}$ & $14^{\mathrm{FGHI}}$ & $22.3^{\mathrm{GHIJK}}$ & $5.32^{\mathrm{JKL}}$ \\
\hline KF170542 & $335^{\mathrm{EFGHI}}$ & $113^{\mathrm{BCD}}$ & $10^{\mathrm{LMN}}$ & $22.1^{\mathrm{GHIJK}}$ & $7.58^{\mathrm{B}}$ \\
\hline KF170485 & $400^{\mathrm{AB}}$ & $95^{\text {JKLMN }}$ & $14^{\mathrm{FGHI}}$ & $20.9^{\mathrm{MNOP}}$ & $6.27^{\mathrm{G}}$ \\
\hline KF170498 & $292^{\text {KLMN }}$ & $89^{\mathrm{MN}}$ & $12^{\mathrm{HIJK}}$ & $24.8^{\mathrm{BC}}$ & $5.99^{\mathrm{GHI}}$ \\
\hline KF170407 & $395^{\mathrm{ABC}}$ & $117^{\mathrm{B}}$ & $11^{\mathrm{JKLM}}$ & $20.9^{\mathrm{MNOP}}$ & $7.03^{\mathrm{CDE}}$ \\
\hline
\end{tabular}




\begin{tabular}{|c|c|c|c|c|c|}
\hline & & & & & \\
\hline KF170509 & $421^{\mathrm{A}}$ & $131^{\mathrm{A}}$ & $9^{\mathrm{LMN}}$ & $22.9^{\mathrm{FGH}}$ & $8.26^{\mathrm{A}}$ \\
\hline KF170390 & $290^{\text {KLMN }}$ & $93^{\mathrm{MN}}$ & $26^{\mathrm{A}}$ & $21.4^{\mathrm{LMNO}}$ & $5.26^{\mathrm{KL}}$ \\
\hline KF170532 & $271^{\mathrm{PQR}}$ & $101^{\mathrm{HIJK}}$ & $14^{\mathrm{FGHI}}$ & $21.6^{\mathrm{IKLLM}}$ & $5.12^{\mathrm{L}}$ \\
\hline KF170502 & $276^{\mathrm{NOPQ}}$ & $98^{\mathrm{HIJKL}}$ & $14^{\mathrm{FGHI}}$ & $21.5^{\mathrm{JLKMN}}$ & $5.16^{\mathrm{L}}$ \\
\hline KF170504 & $255^{\mathrm{QRS}}$ & $95^{\text {JKLMN }}$ & $16^{\mathrm{DEFG}}$ & $21.5^{\text {JKLMN }}$ & $5.4^{\mathrm{JKL}}$ \\
\hline KF170503 & $280^{\mathrm{LMNO}}$ & $106^{\mathrm{CDEF}}$ & $16^{\mathrm{DEFG}}$ & $21.5^{\text {JKLMN }}$ & $5.14^{\mathrm{L}}$ \\
\hline KF170508 & $249^{\mathrm{QRS}}$ & $96^{\mathrm{IJKL}}$ & $17^{\mathrm{DEF}}$ & $24.2^{\mathrm{CD}}$ & $5.42^{\mathrm{JKL}}$ \\
\hline KF170510 & $251^{\mathrm{QRS}}$ & $111^{\mathrm{BCDE}}$ & $8^{\mathrm{NO}}$ & $21.0^{\mathrm{MNOP}}$ & $7.25^{\mathrm{BCD}}$ \\
\hline SAHEL177 & $241^{\mathrm{RS}}$ & $96^{\mathrm{IJKL}}$ & $15^{\mathrm{EFGH}}$ & $24.2^{\mathrm{BCD}}$ & $6.29^{\mathrm{FG}}$ \\
\hline ARICA3 & $356^{\mathrm{DEF}}$ & $113^{\mathrm{BCD}}$ & $13^{\mathrm{HIJ}}$ & $22.3^{\mathrm{GHIJK}}$ & $6.31^{\mathrm{FG}}$ \\
\hline Mean & $317 \pm 4.1$ & $106.6 \pm 0.9$ & $14.4 \pm 0.3$ & $22.5 \pm 0.2$ & $6.5 \pm 0.1$ \\
\hline $\mathrm{CV} \%$ & 10.6 & 6.4 & 11.3 & 3.6 & 6.3 \\
\hline
\end{tabular}

Means followed by the same letter(s) within a column are not significantly different at 0.05 probability level according to LSD Test; Number of panicles/m² (NPM); No. filled grains/panicle (NFGP); No. unfilled grains/panicle (NUFGP); 1000-grain weight (TGW); Grain yield (GY th).

74.5 to $103.0 \mathrm{~cm}$ with the local check (Arica3) as the tallest and genotype KF170489 as the shortest. According to IRRI irrigated rice plant height is classified as semi-dwarf (less than $110 \mathrm{~cm}$ ), intermediate $(110-130 \mathrm{~cm}$ ) and tall (more than $130 \mathrm{~cm}$ ). Based on this classification, in the present study $90.4 \%$ of the tested genotypes group is under the semi-dwarf class. This indicated that the tested genotypes had inherent variability in stature to develop lodging resistant varieties (semi-dwarf) that will have higher response to nitrogen application. Also reported variation in plant height by [19] [20] and [21]. The maximum panicle length value is $25.5 \mathrm{~cm}$ and the minimum is $19.4 \mathrm{~cm}$ for Arica3 and KF170495 respectively. Based on the IRRI irrigated rice classified argument, the present finding showed that there is enough medium variability for panicle length among the genotypes for improving panicle architecture and grain yield due to high association of this trait that determines the number of grains it can hold (Table $3(\mathrm{a}))$. The range for number of filled grain/panicle was 89 to 134 for genotypes KF170505, KF170498 and KF170506 respectively. The range of percentage of unfilled grain/panicle was 8 to 26 for genotypes KF170217, KF170510 and KF170390 respectively (Table 3(b)). Adequate number of fertile grains/ panicle and heavy grains are important traits, which should be considered in selection for high yield genotypes [4] and [19]. Relatively range performance revealed among the studied traits, grain yield (5.12 - $8.35 \mathrm{t} / \mathrm{ha})$. The genotypes were significantly varied for grain yield and about $76.2 \%$ of the genotypes had higher grain yield. Among the genotypes, KF170506, KF170509, KF170542, KF170530, KF170543, KF170500 and KF170510 were the top yielders with grain yield of $8.35,8.26,7.58,7.45,7.40,7.30$ and $7.25 \mathrm{t} / \mathrm{ha}$, respectively. Thus, the existence corresponding of enough variability among genotypes was highly significant. 
Hence, it offers a better scope for further selection of promising DH genotypes in rice breeding program particularly selection based on yield is reliable.

\subsection{Interrelationship}

Yield is a complex product being influenced by several independent quantitative characters. Breeders always look for variation among traits to select desirable types. Some of these characters are highly associated among themselves and with grain yield.

The analysis of the relationships among these characters and their associations with grain yield is essential to establish selection criteria. When more characters are involved in correlation study it becomes difficult to ascertain the characters which really contribute toward yield. The relationships existing between 10 quantitative traits represented as simple correlation coefficients are presented in $\mathrm{Ta}$ ble 4. Correlation of yield and other traits is important in indirect selection for high yield improvement in crop genotypes [22]. There was a highly significant and positive correlation of grain yield with number of filled grain per panicle (0.65), number of tillers per plant (0.64.), number of panicle/ $\mathrm{m}^{2}(0.54)$, panicle length (0.28), 1000 grain weight (0.23) (Table 4). Conversely, grain yield exhibited negative significant correlation with number of unfilled grain per panicle $(-52)$ and days to $50 \%(-0.16)$. The significant and positive correlation with grain yield are a strongly indication that these traits are major factors in improving grain yield; also suggests that selection directed towards these characters will be effective in ensuring high grain yield in doubled haploid rice. These results collaborate with the finding of [23] who observed a positive and significant correlation between grain yield and number of filled gain per panicle. Also, these results were in agreement with that reported of [24] and [25]. The negative correlation obtained between number of unfilled grain per panicle and days to $50 \%$ heading indicate that grain yield can be improved by selecting early maturing genotypes.

Table 4. Simple linear correlation coefficients between 10 pairs of traits in DH rice using (combined over two seasons 2019-2020).

\begin{tabular}{|c|c|c|c|c|c|c|c|c|c|}
\hline Traits & DF & $\mathrm{DM}$ & PLH & PL & NT & NPM & NFGP & NUFG & TGW \\
\hline $\mathrm{DM}$ & $0.60^{* *}$ & & & & & & & & \\
\hline PLH & $0.27^{\star *}$ & $0.15^{\star}$ & & & & & & & \\
\hline PL & -0.029 & 0.05 & $0.23^{\star * *}$ & & & & & & \\
\hline NT & $-0.007^{\star}$ & $0.09^{*}$ & $0.14^{\star}$ & $0.32^{* * *}$ & & & & & \\
\hline NPM & $-0.04^{\star}$ & $-0.04^{\star}$ & $0.23^{\star \star}$ & $0.34^{\star \star}$ & $0.51^{* *}$ & & & & \\
\hline NFGP & $-0.06^{\star}$ & $0.14^{\star}$ & $0.12^{\star}$ & $0.36^{\star \star}$ & $0.58^{\star \star}$ & $0.45^{\star \star}$ & & & \\
\hline NUFG & $0.19^{* *}$ & $0.14^{\star}$ & $-0.10^{*}$ & $-0.20^{\star *}$ & $-0.53^{\star *}$ & $-0.42^{\star *}$ & $-0.43^{* *}$ & & \\
\hline TGW & $-0.26^{\star * *}$ & $-0.09^{\star}$ & -0.01 & $0.11^{\star}$ & $0.11^{\star}$ & $0.08^{\star}$ & $-0.15^{\star}$ & $0.06^{\star}$ & \\
\hline GYT & $-0.16^{*}$ & -0.04 & 0.03 & $0.28^{\star * *}$ & $0.64^{* * *}$ & $0.54^{* * *}$ & $0.65^{* *}$ & $-0.52^{\star *}$ & $0.23^{* *}$ \\
\hline
\end{tabular}

${ }^{*},{ }^{* *}$ and ${ }^{* * *}$ significant at $0.05,0.01$ and 0.001 probability respectively. See footnotes of Table 1 for traits explanations. 


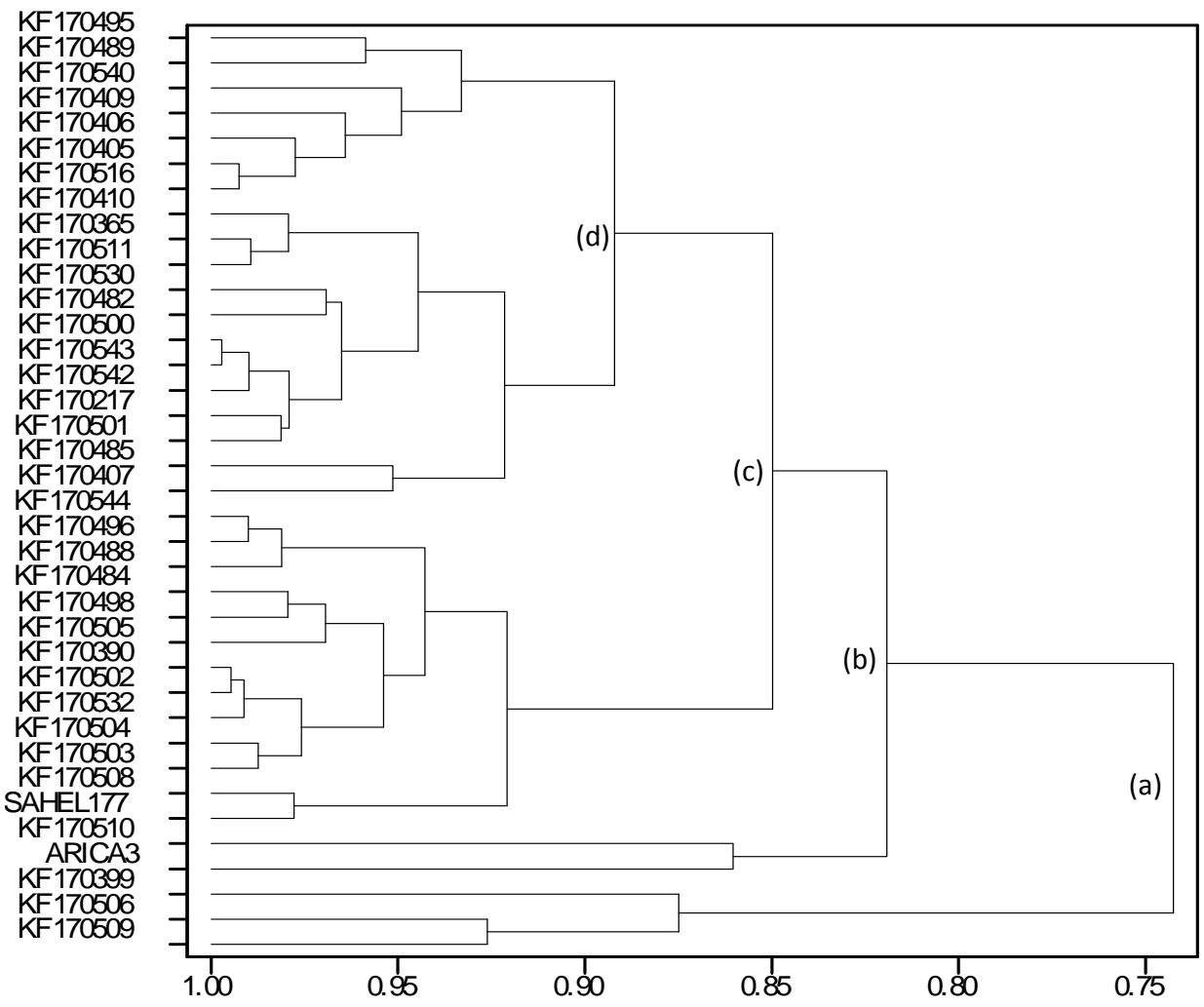

Figure 1. Cluster dendrogram representing distribution of 37rice $\mathrm{DH}$ genotypes based on grain yield components trait.

\subsection{Cluster Analysis}

Among 37 elite rice genotypes, cluster analysis resulted in four clusters (Figure 1). Distribution pattern indicated that maximum genotypes (13) were aggregated in cluster B, while minimum (5) were grouped into cluster A.

Cluster-wise mean values of ten different traits were computed to assess superiority of cluster, which could be considered in the improvement of various characters [26] [27]. Mean performance of different clusters for the characters manifested that genotypes with maximum paddy yield were accumulated in cluster A, whereas genotypes with minimum were in grouped cluster D.

\section{Conclusion}

In conclusion, the present study identified the presence of adequate genetic variability among 37 tested genotypes. Hence, the information generated from this study, rice breeder can be exploited for future rice breeding program. The study was also carried out for one sit and two seasons. Therefore, it is advisable to select and release the highest yielding genotypes (KF170506, KF170509, KF170542, KF170530, KF170543, KF170500 and KF170510) need to check the adaptability and stability with tested at major rice-growing areas to make sound recommendations for release. Moreover, it is recommended that future rice research explore molecular means to further confirm the outcome of DH lines study find- 
ings, in order to do an efficient selection process for quantitative and qualitative traits.

\section{Acknowledgements}

This present study was supported by a grant from Korea-Africa Food and Agriculture Cooperation Initiative (KAFACI), Rural Development Administration (RDA) of Korea; (Project Number: KAR20190115), special recognition for them. Also, thanks forwarded to Africa Rice Saint Louis (Senegal) as a focal point for germplasms exchange among collaborating countries. Also, we highly thank White Nile Research Station staff for spending significant time and effort on data collection and field management.

\section{Conflicts of Interest}

The authors declare no conflicts of interest regarding the publication of this paper.

\section{References}

[1] Bashir, M.U. Akber, N., Iqbal, A. and Zaman, H. (2010) Effect of Different Sowing Dates on Yield and Yield Components of Direct Seeded Coarse Rice (Oryza sativa L.). Pakistan Journal of Agricultural Science, 47, 361-365.

[2] Anyaoha, C., Felix, A., Uyokei, U., Bosede, P., Vernon, G., Semon, M. and Mamadou, F. (2018) Genetic Diversity of Selected Upland Rice Genotypes (Oryza sativa L.) for Grain Yield and Related Traits. International Journal of Plant \& Soil Science, 22, 1-9.

[3] Pang, Y., Chen, K. and Wang, X. (2017) Recurrent Selection Breeding by Dominant male Sterility for Multiple Abiotic Stresses Tolerant Rice Cultivars. Euphytica, 213, Article No. 268. https://doi.org/10.1007/s10681-017-2055-5

[4] Osman, K.A., Mustafa, A.M., Ali, F., Zheng, Y. and Qiu, F. (2012) Genetic Variability for Yield and Related Attributes of Upland Rice Genotypes in Semi Arid Zone (Sudan). African Journal of Agricultural Research, 7, 4613-4619. https://doi.org/10.5897/AJAR12.529

[5] Ali, J., Xu, J.L., Gao, Y.M., Ma, X.F., Meng, L.J., Wang, Y., Pang, Y.L., Guan, Y.S., Xu, M.R., Revilleza, J.E., Franje, N.J., Zhou, S.C. and Li, Z.K. (2017) Harnessing the Hidden Genetic Diversity for Improving Multiple Abiotic Stress Tolerance in Rice (Oryza sativa L.). PLoS ONE, 12, e0172515. https://doi.org/10.1371/journal.pone.0172515

[6] Fischer, R.A. (2015) Definitions and Determination of Crop Yield, Yield Gaps, and of Rates of Change. Field Crops Research, 182, 9-18. https://doi.org/10.1016/j.fcr.2014.12.006

[7] Dawson, I.K., Russell, J., Powell, W., Steffenson, B., Thomas, W.T.B. and Waugh, R. (2015) Barley: A Translational Model for Adaptation to Climate Change. New Phytologist, 206, 913-931. https://doi.org/10.1111/nph.13266

[8] Dwivedi, S.L., Britt, A.B., Tripathi, L., Sharma, S., Upadhyaya, H.D. and Ortiz, R. (2015) Haploids: Constraints and Opportunities in Plant Breeding. Biotechnology Advances, 33, 812-829. https://doi.org/10.1016/j.biotechadv.2015.07.001

[9] Herawati, R., Purwoko, B.S. and Dewi, I.S. (2010) Characterization of Doubled 
Haploid Derived from Anther Culture for New Type Upland Rice. Jurnal Agronomi Indonesia, 38, 170-176.

[10] Roy, B. and Mandal, A.B. (2005) Anther Culture Response in Indica and Variations in Major Agronomic Characters among the Androclones of a Scented Cultivar, Karnal Local. African Journal of Biotechnology, 4, 235-240.

[11] Seguí-Simarro, J.M. (2015) Editorial: Doubled Haploid in Model and Recalcitrant Species. Frontiers in Plant Science, 6, Article No. 1175.

https://doi.org/10.3389/fpls.2015.01175

[12] Khush, G.S. and Brar, D.S. (2002) Biotechnology for Rice Breeding: Progress and Impact. Proceedings of the 20 th Session of the International Rice Commission, Bangkok, 23-26 July 2002, 41-58.

[13] IRRI (2002) Standard Evaluation System for Rice. International Rice Research Institute (IRRI), Manila.

http://www.knowledgebank.irri.org/images/docs/rice-standard-evaluation-system.pdf

[14] SAS Institute (2000) SAS Linear Model: A Guide to ANOVA and GLM Procedures. SAS Inst. Inc., Cary, NC.

[15] Singh, R.K. and Chaudhary, B.D. (1985) Biometrical Methods in Quantitative Genetic Analysis. 3rd Edition, Kalyani Publisher, New Delhi.

[16] Sohrabi, M., Rafii, M., Hanafi, M.M. Akmar, A.S. and Latif, M.A. (2012) Genetic Diversity of Upland Rice Germplasm in Malaysia Based On Quantitative Traits. The Scientific World Journal, 2012, Article ID: 416291. https://doi.org/10.1100/2012/416291

[17] Ahmed, T., Kumar, A., Pandey, D. and Prasad, B. (2018) Correlation and Path Coefficient Analysis for Yield and Its Attributing Traits in Bread Wheat (Triticum aestivum L.). Journal of Applied and Natural Science, 10, 1078-1084.

[18] Mulugeta, S., Sentayehu, A. and Kassahun, B. (2011) Evaluation of Upland NERICA Rice (Oryza sativa L.) Genotype for Grain Yield and Yield Components along an Altitude Gradient in Southwest Ethiopia. Journal of Agronomy, 10, 105-111.

[19] Abebe, T., Alamerew, S. and Tulu, L. (2017) Genetic Variability, Heritability and Genetic Advance for Yield and its Related Traits in Rainfed Lowland Rice (Oryza sativa L.) Genotypes at Fogera and Pawe, Ethiopia. Advances in Crop Science and Technology, 5, Article No. 272. https://doi.org/10.4172/2329-8863.1000272

[20] Akinwale, M., Gregorio, G., Nwilene, F., Akinyele, B., Ogunbayo, S.A. and Odiyi, A.C. (2011) Heritability and Correlation Coefficient Analysis for Yield and Its Components in Rice (Oryza sativa L.). African Journal of Plant Science, 5, 207-212.

[21] Idris, A.E. and Mohamed, K.A. (2013) Estimation of Genetic Variability and Correlation for Grain Yield Components in Rice (Oryza sativa L.). Global Journal of Plant Ecophysiology, 3, 1-6.

[22] Idris, A.E., Justin, F.J., Dagash, Y.M. and Abuali, A.I. (2012) Genetic Variability and Inter Relationship between Yield and Yield Components in Some Rice Genotypes. American Journal of Experimental Agriculture, 2, 233-239.

[23] Ogunbayo, S.A., Sie, M., Ojo, D.K., Sanni, K.A., Akinwale, M.G., Toulou, B., Shuttu, A., Idehen, E.O., Popoola, A.R., Daniel, I.O. and Gregorio, G.B. (2014) Genetic Variation and Heritability of Yield and Related Traits in Promising Rice Genotypes (Oryza sativa L.). Asian Journal of Plant Sciences, 6, 653-666.

[24] Kato, Y., Kamoshita, A. and Yamagishi, J. (2008) Preflowering Abortion Reduces Spikelet Number in Upland Rice (Oryza sativa L.) under Water Stress. Crop Science, 48, 2389-2395. https://doi.org/10.2135/cropsci2007.11.0627 
[25] Surek, H. and Beser, N. (2003) Correlation and Path Coefficient Analysis for Some Yield Relatedtriats in Rice (Oryza sativa L.). Crop Research, 12, 165-167.

[26] Tuhina, K.M., Mohamed, M.H, Mohd, R.Y., Wong, M.Y. Faezah, M.S. and Jannatul, F. (2015) Genetic Variation, Heritability, and Diversity Analysis of Upland Rice (Oryza sativa L.) Genotypes Based on Quantitative Traits. BioMed Research International, 2015, Article ID: 290861. https://doi.org/10.1155/2015/290861

[27] Maxwell, D.A., Adjah, K.L. and Ebenezer, A.A. (2019) Assessment of Genetic Diversity for Grain Yield and Yield Component Traits in Some Genotypes of Rice (Oryza sativa L.). Journal of Crop Science and Biotechnology, 22, 123-130.

https://doi.org/10.1007/s12892-019-0008-0 